DOI: $10.4274 /$ jarem.galenos.2020.3185

J Acad Res Med 2020;10(3):237-40

\title{
Comparison and Analyses of Intraoperative Consultation and Paraffin Section Responses of Ovarian Lesions
}

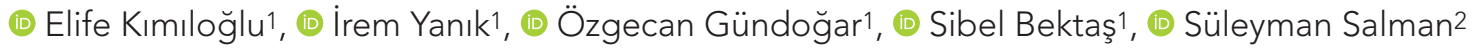 \\ ${ }^{1}$ University of Health Sciences Turkey, Gaziosmanpaşa Health Practice and Research Center, Department of Medical Pathology, İstanbul, \\ Turkey \\ 2University of Health Sciences Turkey, Gaziosmanpaşa Health Practice and Research Center, Department of Gynecology and Obstetrics, \\ İstanbul, Turkey
}

Cite this article as: Kımıloğlu E, Yanık İ, Gündoğar Ö, Bektaş S, Salman S, Comparison and Analyses of Intraoperative Consultation and Paraffin Section Responses of Ovarian Lesions. J Acad Res Med 2020;10(3):237-40

\begin{abstract}
Objective: We aimed to analyze the sensitivity, specificity, positive predictive values (PPV) and negative predictive values (NPV) and the accuracy of frozen section (FS) method and compare the concordance of FS responses and paraffin section (PS) (final histopathological diagnoses) results of the ovarian mass operations in our hospital.

Methods: We retrospectively reviewed the cases of ovarian lesions operated between March 2008 and June 2018 in which FS was requested. In total 205 cases were found and the results were compared. The FS responses and PS results were classified as benign, borderline and malignant. Seventeen cases weren't responded in FS (and left to be diagnosed in PS) and they were left out of the study.

Results: According to the analyses; the sensitivity of benign, borderline and malignant tumors were $100 \%, 83 \%$, $95 \%$, and the specificity were $98 \%$, $99 \%, 98 \%$, respectively. The PPV of borderline and malignant tumors were $91 \%, 92 \%$ and the NPV were $98 \%, 99 \%$ respectively. Furthermore; both of their accuracy was $97 \%$. The concordance between the methods was assessed with Kappa test and found as $94 \%(p<0.001)$.

Conclusion: In our study we showed that even if all of the FS responses didn't match with its PS, FS has a very high rate of consistency. FS is a way to help the surgeon during the operation. However, it must not be forgotten that this method has its own pitfalls and limitations.

Keywords: Ovary, frozen section, paraffin section, diagnosis accuracy
\end{abstract}

\section{INTRODUCTION}

Intraoperative consultation, or more commonly referred as frozen section (FS), plays a crucial part in pathology. FS is especially important during ovarian mass surgeries since radiological imaging and biochemical markers are limited in determining these lesions' malignancy. Intraoperative consultations are performed to help operating surgeons decide the surgical approach.
This study intended to evaluate the concordance of FS responses and paraffin section (PS) (final histopathological diagnoses) results of ovarian neoplasm surgeries conducted in our hospital and to determine the sensitivity, specificity, positive predictive values (PPV) and negative predictive values (NPV), and accuracy of FS in categorizing ovarian lesions as benign, borderline, and malignant.

ORCID IDs of the authors: E.K. 000000022708 1272; I.Y. 0000-0001-6227-1189; Ö.G. 000000033075 6063; S.B. 0000-0003-0248-9869; S.S. 0000 000170906105 . 


\section{METHODS}

\section{Subjects}

The study retrospectively reviewed cases of ovarian neoplasms operated in our hospital between March 2008 and June 2018 in which intraoperative pathology consultation (FS) were done. A total of 205 cases were included. The reports of the pathologists, who were working in our department during this time, were reviewed, and the FS responses and final PS results were grouped accordingly. PS results were grouped as benign (both neoplastic and non-neoplastic lesions), borderline, and malignant (including metastasis cases), and FS responses were grouped as benign, borderline (including "at least borderline" responses), and malignant. Juvenile and adult granulosa cell tumors, which are considered "low potential of malignancy," were included in the borderline group (1).

Seventeen cases with no definitive FS responses were excluded from this study as they have no equivalent PS results.

The study was approved by the Taksim Training and Research Hospital, Clinic Research Ethics Committee (approval number: 89, date: 19.09.2018).

\section{Statistical Analysis}

Data were presented as frequency and percentage, and concordance between the methods was evaluated using the Kappa test. Sensitivity, specificity, PPV, NPV, accuracy, and 95\% confidence interval were determined. The limit of significance was set at $p<0.05$ and bidirectional. Analyses were conducted using the NCSS 10 program (Kaysville, UT, USA).

\section{RESULTS}

During FS, 127 cases were identified as benign, 22 borderline, and 39 malignant. Of the cases, 17 had a definitive response in FS for varying reasons, and they were left to be determined in PS and, therefore, excluded from this study.

\section{Table 1. Numerical distribution of cases}

\begin{tabular}{|c|c|c|c|}
\hline \multirow{2}{*}{ Benign } & Non-neoplastic & $62(33 \%)$ & \multirow{2}{*}{$126(67 \%)$} \\
\hline & Neoplastic & 64 (34\%) & \\
\hline Borderline & - & - & 24 (13\%) \\
\hline Malignant & Metastases & $6(3 \%)$ & $38(20 \%)$ \\
\hline \multicolumn{2}{|l|}{ Total } & \multicolumn{2}{|l|}{188 (100\%) } \\
\hline
\end{tabular}

Table 2. Numerical comparison between paraffin and intraoperative consultation result

\begin{tabular}{|c|c|c|c|c|c|}
\hline & & \multicolumn{3}{|c|}{ Paraffin results } & \multirow{2}{*}{ Total } \\
\hline & & Benign & Borderline & Malignant & \\
\hline \multirow{3}{*}{ Intraoperative consultation results } & Benign & 126 & 1 & 0 & 127 \\
\hline & Borderline & 0 & 20 & 2 & 22 \\
\hline & Malignant & 0 & 3 & 36 & 39 \\
\hline Total & & 126 & 24 & 38 & 188 \\
\hline
\end{tabular}

PS results showed that there were 126 (67\%) benign cases: 62 (33\%) non-neoplastic and 64 (34\%) neoplastic. Furthermore, 24 (13\%) cases were reported as borderline. Finally, 38 cases were diagnosed as malignant, of which $33(16 \%)$ were primary ovarian tumors and 7 (4\%) metastases (Table 1).

Of the 17 cases that were left to be determined in PS, 12 were benign, 3 borderline, and 2 malignant.

The PS and FS responses were compared numerically (Table 2), and 1 of the 127 benign cases in FS was later reported as borderline, 2 of the 22 borderline cases were later reported as malignant, and 3 of the 39 malignant cases were later diagnosed as borderline.

In this study, the mean age of all 188 cases was 49.5 years. When all cases were reviewed, 182 of 188 cases, for which a FS response was given, were compatible with the final paraffin diagnoses, and the outcome showed a consistency of $96.8 \%$.

According to statistical analyses (Table 3), the sensitivities of FS responses of benign, borderline, and malignant tumors were $100 \%, 83 \%$, and $95 \%$, and the specificity results were $98 \%, 99 \%$, and $98 \%$, respectively. The PPVs of borderline and malignant tumors were $91 \%$ and $92 \%$, and NPVs were $98 \%$ and $99 \%$, respectively. Also, both tumors have an accuracy of $97 \%$. The concordance between the methods was evaluated using the Kappa test and was $94 \% \pm 0.03 \%(p<0.001)$.

\section{DISCUSSION}

Intraoperative pathology consultation (or FS) is an essential method that must be done meticulously since it helps surgeons perform optimal operations for patients. The FS method preserves its significance because of non-specific biomarkers, which also frequently result in false positive or false negative results, and limited preoperative radiological imaging in ovarian neoplasm adequacy. Overdiagnosis during FS will extend the operation time, which may increase mortality and morbidity, 
Table 3. Statistical analyses of cases classified as benign, borderline, and malignant

\begin{tabular}{|l|l|l|l|}
\hline & Benign & Borderline & Malignant \\
\hline Sensitivity (95\% GA) & $1(0.97-1)$ & $0.83(0.63-0.95)$ & $0.95(0.82-0.99)$ \\
\hline Specificity (95\% GA) & $0.98(0.91-1)$ & $0.99(0.96-1)$ & $0.98(0.94-1)$ \\
\hline PPV (95\% GA) & $0.99(0.95-1)$ & $0.91(0.71-0.98)$ & $0.92(0.80-0.97)$ \\
\hline NPV (95\% GA) & $1(0.92-1)$ & $0.98(0.94-0.99)$ & $0.99(0.95-1)$ \\
\hline Accuracy (95\% GA) & $0.99(0.97-1)$ & $0.97(0.93-0.99)$ & $0.97(0.94-0.99)$ \\
\hline PPV: positive predictive value, NPV: negative predictive value & & \\
\hline
\end{tabular}

whereas underdiagnosis may require reoperation or cause tumor to spread (2).

Cases that were inconsistent with PS were reviewed, and it was found that errors might have occurred in some steps. The intraoperative consultation process consists of some crucial parts, including proper macroscopic examination and appropriate sampling. Errors in these parts could be avoided by proper training and experience. Tissue freezing can also result in frozen/ freezing artifacts, which might cause the cells to look different than in formalin-fixed tissue samples, thus completely altering the diagnoses. Another important step is the staining part, and errors here might cause the nuclei to appear more hyperchromatic than they are. After meticulously performing all these steps, the pathologists should provide a result in the shortest possible time.

In our study, the group classified as benign contained both non-neoplastic and neoplastic lesions. Endometriosis, follicle cysts, corpus luteum cysts, and abscesses were considered nonneoplastic, whereas cystadenomas, cystadenofibromas, mature cystic teratomas, fibromas, thecomas, and fibrothecomas were neoplastic. During FS, the pathologists did not provide a result (left it to be decided in PS) for some of these benign lesions, such as fibromas/thecomas/fibrothecomas.

Of the 127 cases, which were considered benign during intraoperative consultation, only one did not correlate with the final PS report. During consultation, the case appeared "benign" on FS slides and was thought to be a sex-cord stromal tumor. After 24 hours of formalin fixation, more samples were taken. All the PS slides were evaluated both morphologically and immunohistochemically. The final diagnosis was "adult granulosa cell tumor," which is considered "low malignant potential," and in our study, it is classified as borderline tumors.

The correlation in the benign group was $99.2 \%$. Similar to our study, other studies also have high sensitivity to benign lesions: Sukumaran et al. (2) reported $99.2 \%$, Bige et al. (3) $99.2 \%$, and Arshad et al. (5) 95.6\% (2-6).

Borderline tumors make up close to $15 \%-20 \%$ of all ovarian malignancies (7). Borderline epithelial tumors and some sex-cordstromal tumors (which are considered low/unknown malignant potential tumors like adult/juvenile granulosa cell tumors) were included in the borderline group in our study. The borderline group had the lowest concordance and sensitivity rate at $90.9 \%$ and $83 \%$, respectively. Similar sensitivity results were reported in other studies: $88.46 \%$ (3), $77.8 \%$ (4) and $76.2 \%$ (5), thus supporting our findings.

During intraoperative consultations, 2 of 22 border lesions were later categorized as malignant in PS. These tumors were large, and only a limited amount of sampling was done from the areas most suspicious for invasion. No evidence of invasion was found in the frozen slides; however, suspicion of malignancy remained. Therefore, they were reported as "at least borderline" during intraoperative consultation. More samplings were performed from macroscopically detected suspicious thickening of the lesion wall and from the papillary appearing areas after the 24 hours formalin fixation. Routine staining limited the number of invasive areas identified, and therefore, these tumors were later reported as malignant during PS.

The remaining 20 cases were compatible. The two cases, which the pathologists classified FS as "at least borderline" may be statistically seen as an error. However, the surgeons knew the possibility of them being malignant during the operation, and they proceeded accordingly. Since sampling problems during FS for borderline tumors are widely known, the term "at least borderline" as a response is supported by other authors (8).

Tempfer et al. (9) collected data from four studies (including their own) and evaluated borderline tumors during FS. Results showed that consistency between FS and PS was observed in $62.8 \%$ of the cases. According to them, because of this high ratio of underdiagnosis/overdiagnosis (37.2\%), when FS is classified as borderline, surgeons should not take any further action during the operation and wait for PS reports. It is also suggested that for larger lesions $(>5 \mathrm{~cm})$, after a thorough macroscopic evaluation, multiple samples might be used to answer FS or intraoperative consultation might not be done at all (9).

An additional observation showed that subspecialization of pathologists in gynecologic pathology yielded better results (9). For example, Bige et al. (3) presented two sets of data from gynecologic and non-gynecologic pathologists and found that percentages of sensitivity, specificity, PPV and NPV were higher in gynecologic pathologists, especially in the borderline group. According to Açikalin et al. (4), gross examination conducted by gynecologic pathologists during FS is an important factor to increase FS accuracy. This factor might be one of the limitations of our study because our department has no subspecialized pathologists. 
Other reasons that borderline tumors are challenging to assess during FS include their heterogeneous component and size. Only a limited amount of sampling can be done in FS; however, after fixation, more lesions can be sampled, and this may very well change the diagnosis $(2,6,10)$.

In the malignant group, there were two cases of primary ovarian tumors and metastases. In 36 of the 39 malignant cases, the FS responses and PS results were consistent. However, the remaining three cases were later reported as borderline lesions because the cells that were considered invasive were actually pseudoinvasions and the nuclei appeared more hyperchromatic because of staining and improper sectioning (such as section thickness).

The concordance rate and sensitivity of the malignant group were $92.3 \%$ and $95 \%$, respectively. Açikalin et al. (4) and Bige et al. (3) had sensitivities of $95.6 \%$ and $95 \%$, respectively, which are similar to our study. However, Sukumaran et al. (2) had a slightly lower sensitivity (82.95\%), and Takemoto et al. (10) had slightly higher data (99.2\%).

According to statistical findings, a high consistency was found between these methods (Kappa 0.94, p<0.001). The benign lesion group had the highest sensitivity and accuracy rates (100\% and $99 \%$, respectively), whereas the borderline group had the highest specificity (99\%).

\section{CONCLUSION}

Although the coherency between FS and PS is not 100\%, it is still high, and that is why FS still plays a crucial part in pathology practice. Considering these findings, surgeons must not forget that FS might be limited and have pitfalls; therefore, they should plan their operations accordingly.

Ethics Committee Approval: The study was approved by the Taksim Training and Research Hospital, Clinic Research Ethics Committee (approval number: 89, date: 19.09.2018).
Informed Consent: Retrospective study.

Peer-review: Externally peer-reviewed.

Author Contributions: Concept - E.K., Design - I.Y., Supervision - E.K., S.S., Resources - E.K., S.B., S.S., Materials - S.S., Data Collection and/or Processing - I.Y., Ö.G., Analysis and/or Interpretation - I.Y., Ö.G., Literature Search - S.B., Writing Manuscript - I.Y., Ö.G., Critical Review - E.K., S.B.

Conflict of Interest: The authors have no conflict of interest to declare.

Financial Disclosure: The authors declared that this study has received no financial support.

\section{REFERENCES}

1. Kurman RJ, Carcangiu ML, Herrington CS, Young RHE, editors. WHO classification of tumours of female reproductive organs. IARC: Lyon; 2014.

2. Sukumaran R, Somanathan T, Mathews A, Kattor J, Sambasivan S, Nair RP. Role of frozen section in intraoperative assessment of ovarian masses: a tertiary oncology center experience. Indian J Surg Oncol 2014; 5: 99103.

3. Bige O, Demir A, Saygili U, Gode F, Uslu T, Koyuncuoglu M. Frozen section diagnoses of 578 ovarian tumors made by pathologists with and without expertise on gynecologic pathology. Gynecol Oncol 2011; 123: 43-6.

4. Açikalin A, Torun G, Bağir E, Bayram F, Zeren H, Güleç U, et al. Intraoperative frozen section in ovarian neoplasms; a tertiary center experience. Turk Patoloji Derg 2014; 30: 184-8.

5. Arshad NZ, Ng BK, Paiman NA, Abdullah Mahdy Z, Mohd Noor R. Intra-operative frozen sections for ovarian tumors - a tertiary center experience. Asian Pac J Cancer Prev 2017; 19: 213-8.

6. Hashmi AA, Naz S, Edhi MM, Faridi N, Hussain SD, Mumtaz S, et al. Accuracy of intraoperative frozen section for the evaluation of ovarian neoplasms: an institutional experience. World J Surg Oncol 2016; 14: 91.

7. Seong SJ, Kim DH, Kim MK, Song T. Controversies in borderline ovarian tumors. J Gynecol Oncol 2015; 26: 343-9.

8. Shih KK, Garg K, Soslow RA, Chi DS, Abu-Rustum NR, Barakat RR. Accuracy of frozen section diagnosis of ovarian borderline tumor. Gynecol Oncol 2011; 123: 517-21.

9. Tempfer CB, Polterauer S, Bentz EK, Reinthaller A, Hefler LA. Accuracy of intraoperative frozen section analysis in borderline tumors of the ovary: a retrospective analysis of 96 cases and review of the literature. Gynecol Oncol 2007; 107: 248-52.

10. Takemoto S, Ushijima K, Kawano R, Fukui A, Terada A, Fujimoto T, et al. Validity of intraoperative diagnosis at laparoscopic surgery for ovarian tumors. J Minim Invasive Gynecol 2014; 21: 576-9. 